\title{
Periplocin Extracted from Cortex Periplocae Induced Apoptosis of Gastric Cancer Cells via the ERK1/2-EGR1 Pathway
}

\author{
Lei Li Lian-Mei Zhao Su-li Dai Wen-Xuan Cui Hui-Lai Lv Liang Chen \\ Bao-En Shan \\ Research Centre, the Fourth Hospital of Hebei Medical University, Shijiazhuang, China
}

\section{Key Words}

Periplocin • Apoptosis • Gastric cancer cell • EGR1

\begin{abstract}
Background/Aims: Periplocin is extracted from the traditional herbal medicine cortex periplocae, which has been reported to suppress the growth of cancer cells. However, little is known about its effect on gastric cancer cells. Methods: Gastric cancer cells were treated with periplocin, and cell viability was assessed using MTS assay. Flow cytometry and TUNEL staining were performed to evaluate apoptosis, and protein expression was examined by western blotting. Microarray analysis was used to screen for changes in related genes. Results: We found that periplocin had an inhibitory effect on gastric cancer cell viability in a dose-dependent manner. Periplocin inhibited cell viability via the ERK1/2-EGR1 pathway to induce apoptosis. Periplocin also inhibited the growth of tumor xenografts and induced apoptosis in vivo. Conclusion: Our results show that periplocin inhibits the proliferation of gastric cancer cells and induces apoptosis in vitro and in vivo, indicating its potential to be used as an antitumor drug.
\end{abstract}

\section{(c) 2016 The Author(s)}

Published by S. Karger AG, Base

\section{Introduction}

Gastric cancer is the fourth most common cancer and the second most common cause of cancer deaths worldwide and is particularly prevalent in Asian countries [1]. Currently, an effective treatment for the disease is not available, and identification of early stage gastric cancer is difficult given that it is often asymptotic or misdiagnosed [2]. Surgery is the chief prevailing therapy for gastric cancer, but $42.2 \%$ of patients with advanced gastric cancer are unable to undergo surgical therapy [3]. While chemotherapy is also an option for treatment

L. Li and L.-M. Zhao contributed equally to this work. 


\section{Cellular Physiology Cell Physiol Biochem 2016;38:1939-1951 \begin{tabular}{c|l} 
DOI: 10.1159/000445555 & $\begin{array}{l}\text { O 2016 The Author(s). Published by S. Karger AG, Basel } \\
\text { www.karger.com/cpb }\end{array}$ \\
\hline
\end{tabular}}

Li et al.: Periplocin Induced Apoptosis via ERK1/2-EGR1

of gastric cancer, it is likely to have off-target effects that can damage other tissues and organs as well [4]. Therefore, it is imperative to develop novel and effective chemotherapeutic agents that target critical pathways and induce the death of gastric cancer cells.

Cortex periplocae is the dry root of Periploca sepium Bge. It is a traditional herbal medicine that contains a variety of compounds, such as sterols, glycosides and esters, and can be used in cardiotonic and diuretic treatments [5]. Itokawa et al., initially found that periplocoside A from cortex periplocae inhibited the proliferation of S180 cancer cells [6]. Recent reports have shown that periplocin extracted from cortex periplocae significantly inhibits the growth of several tumor cells, including colon cancer and lung cancer cells, and promotes the induction of the apoptosis signaling pathway $[7,8]$. It also exhibits low toxicity in normal cells. However, the complex mechanism by which periplocin renders its antitumor activity has not been elucidated. While studies have shown that periplocin inhibits the proliferation of colon cancer or esophageal cancer cells by inducing apoptosis, the effects of cortex periplocae in gastric cancer have not been examined yet. Therefore, the purpose of our study was to investigate the effect of cortex periplocae on gastric cancer cells and to determine its molecular mechanism.

\section{Materials and Methods}

\section{Cell lines and cell culture}

Human gastric cancer cell lines SGC-7901, MGC-803 and BGC-823 and the normal epithelial gastric cell line GES-1 were obtained from the Cellular Biology Institute of the Shanghai Academy of Sciences (Shanghai, China) and cultured in RPMI-1640 medium containing 10\% bovine serum, penicillin (100 U/ml) and streptomycin $(100 \mathrm{\mu g} / \mathrm{ml})$ at $37^{\circ} \mathrm{C}$ in a $5 \% \mathrm{CO}_{2}$ atmosphere.

\section{Chemicals and antibodies}

Periplocin (purity $\geq 96 \%$ ) was obtained from the New Drug Research and Development Center of North China Pharmaceutical Group Corporation (Hebei, China). It was dissolved in DMSO and diluted to 50, $100,200 \mathrm{ng} / \mathrm{ml}$ using RPMI-1640 medium (the final concentration of DMSO < $0.01 \%$ ). Fetal bovine serum was purchased from Biological Industries (Beit Haemek, Israel). RPMI-1640 medium was purchased from Gibco-BRL (Life Technologies, Paisley, Auckland). Antibodies against Mcl-1, Bid, p-ERK1/2, ERK1/2 and Ki-67 were purchased from Cell Signaling Technology (CST, CA, USA), caspase-3, EGR1 and GAPDH were from Abcam (Cambridge, UK). DeadEnd ${ }^{\mathrm{TM} C}$ Colorimetric TUNEL System and 3-(4, 5-dimethylthiazol-2-yl)5-(3-carboxymethoxyphenyl)-2-(4-sulfophenyl)-2H-tetrazolium, inner salt (MTS) were purchased from Promega Corporation (Madison, USA). Annexin V FITC Apoptosis Kit was purchased from BD Biosciences (San Diego, CA, USA). SPlink Detection Kits and 3, 3'-diaminobenzidine tetrahydrochloride were purchased from ZSGB-BIO (Beijing, China). siRNA Transfection Reagent Kit was purchased from RIBOBIO (Guangzhou, China).

MTS assay detects periplocin-mediated inhibition of growth in human gastric cancer cell lines (SGC7901, MGC-803, BGC-823 and GES-1)

Cells in the logarithmic growth phase were plated in 96-well culture plates at $1 \times 10^{4}$ cells/well. Upon treatment of cells with periplocin $(0,50,100,200 \mathrm{ng} / \mathrm{ml})$ for $24 \mathrm{~h}$ and $48 \mathrm{~h}, 20 \mu \mathrm{l}$ of MTS solution was added to each well, followed by a $2 \mathrm{~h}$ MTS incubation. Then, the optical densities (ODs) were measured using a microplate reader at $490 \mathrm{~nm}$. The cell growth inhibition rate was calculated using the following formula: $1-\mathrm{OD}$ /OD

Flow cytometry analysis of apoptosis with Annexin-V/PI double staining

SGC-7901 and MGC-803 cells were treated with periplocin $(0,50,100,200 \mathrm{ng} / \mathrm{ml})$ for $24 \mathrm{~h}$. Approximately $5 \times 10^{5}$ cells were harvested and washed twice with phosphate buffer saline (PBS) followed by resuspension in $500 \mu \mathrm{l}$ of $1 \mathrm{X}$ binding buffer. Five microliters Annexin V and $5 \mu \mathrm{l}$ PI were added to the solution and incubated for 5 minutes at room temperature in the dark. The cells were gently vortexed for flow cytometric analysis. 


\section{Cellular Physiology Cell Physiol Biochem 2016;38:1939-1951 \begin{tabular}{l|l} 
and Biochemistry Published online: May 09, 2016 & $\begin{array}{l}\text { D } 2016 \text { The Author(s). Published by S. Karger AG, Basel } \\
\text { www.karger.com/cpb }\end{array}$ \\
\hline
\end{tabular}}

Li et al.: Periplocin Induced Apoptosis via ERK1/2-EGR1

Western blot analysis

Proteins were extracted with RIPA buffer containing protease inhibitor, and the concentrations were determined using BCA assay. Equal amounts of protein $(50 \mu \mathrm{g})$ from each sample was separated by $12 \%$ sodium dodecyl sulfate polyacrylamide gel (SDS-PAGE) and transferred to a polyvinylidene difluoride (PVDF) membrane (Millipore), which was then incubated for $1 \mathrm{~h}$ at room temperature with 5\% skim milk. Afterwards, the membranes were incubated overnight with appropriate primary antibodies at $4{ }^{\circ} \mathrm{C}$. After washing the membranes three times with tris buffered saline containing Tween (TBST), they were incubated with fluorochrome-labeled secondary anti-rabbit IgG (IRDye 800-LI-COR, Odyssey) for $1 \mathrm{~h}$ at room temperature. Membranes were imaged using the Odyssey infrared imaging system (USA, LI-COR). GAPDH was taken as a loading control. Western blot analysis was repeated independently 3 times.

Terminal deoxynucleotidyl transferase (TdT) - mediated dUTP nick end labeling (TUNEL) assay for cell apoptosis

The DeadEnd ${ }^{\mathrm{TM}}$ Colorimetric TUNEL System was used to assay cell apoptosis. To detect apoptosis in cultured cells, coverslips were immersed in 4\% paraformaldehyde for 25 min, 0.2\% Triton X-100 in PBS for $5 \mathrm{~min}$, and $100 \mu \mathrm{l}$ Equilibration Buffer at room temperature for $5 \mathrm{~min}$, followed by addition of $100 \mu \mathrm{l}$ TdT reaction mix ( $98 \mu \mathrm{l}$ Equilibration Buffer, $1 \mu \mathrm{l}$ Biotinylated Nucleotide Mix and $1 \mu \mathrm{l}$ rTdT Enzyme) and incubation at $37^{\circ} \mathrm{C}$ for $60 \mathrm{~min}$. The coverslips were then immersed in $2 \times \mathrm{SSC}$ for $15 \mathrm{~min}, 0.3 \%$ hydrogen peroxide for $3 \mathrm{~min}$, and $100 \mu \mathrm{l}$ Streptavidin HRP for $30 \mathrm{~min}$, after which $100 \mu \mathrm{l}$ DAB was added until a light brown background developed. For detection of apoptosis in tissue sections, paraffin-embedded tissue sections were deparaffinized and permeabilized with Proteinase K. The other steps were similar to apoptosis detection in cultured cells. The nuclei of apoptotic cells were stained dark brown. Quantitative analysis was performed blindly by counting the number of TUNEL-positive cells in ten microscopic fields, as described previously. Apoptosis rate $\%=$ (apoptotic cells/total cells) $\%$.

\section{Microarray data}

Differential gene expression between SGC-7901 cells treated with $100 \mathrm{ng} / \mathrm{ml}$ periplocin and untreated cells was analyzed. Total RNA of cells was extracted using TRIzol reagent. The Affymetrix Human Genome U133 Plus 2.0 Array was used to detect gene expression profiles following the manufacturer's instructions. Preliminary data analysis was conducted using the software of the Affymetrix microarray suite. The cutoff criterion for gene calls was at least a 4-fold difference in expression between two compared profiles, and p-values $<0.05$ were identified. The microarray data set was submitted to the GEO repository (GSE78211).

\section{Cell rescue}

The level of EGR1 in SGC-7901 cells was knocked down using the human-specific EGR1 siRNA Transfection Reagent Kit following the manufacturer's protocol. Effective downregulation of EGR1 in cell lines was verified using RT-PCR. First, SGC-7901 cells were treated with periplocin $18 \mathrm{~h}$. Second, the EGR1 siRNA or negative control with transfection reagents were overlaid on the cells for $6 \mathrm{~h}$ at $37^{\circ} \mathrm{C}$ in a $5 \% \mathrm{CO}_{2}$ atmosphere. Finally, cell viability was measured by MTS assay after transfer of cells into normal growth medium for $24 \mathrm{~h}$.

\section{Tumor xenograft study using athymic nude mice}

To determine the in vivo chemotherapeutic efficacy of periplocin against gastric cancer tumor xenogr aft growth, five- to six-week-old male immunodeficient BALB/c mice $(n=12)$ were obtained from Beijing Weitong Lihua Experimental Animal Technology Co. Ltd (Beijing, China). The animals were housed at the Fourth Hospital of Hebei Medical University Experiment Animal Center. SGC-7901 cells $\left(1 \times 10^{5} / 100 \mu \mathrm{l} /\right.$ mouse) were suspended in PBS and injected subcutaneously into the right flank of each mouse. The mice were randomized into four groups. Group I $(\mathrm{n}=3)$ was treated with $0.9 \%$ physiological saline; Group II (n = 3) was treated with $0.04 \mathrm{mg}$ cisplatin; Group III $(\mathrm{n}=3)$ was treated with $0.36 \mathrm{mg}$ periplocin; and Group IV ( $\mathrm{n}=3$ ) was treated with $0.09 \mathrm{mg}$ periplocin every two days. The tumor sizes in the mice in each group were measured every two days. After 14 days, the mice were sacrificed and the tumors were harvested. The tumor tissues were fixed in formalin to obtain sections for the TUNEL assay and immunohistochemistry. 


\section{Cellular Physiology Cell Physiol Biochem 2016;38:1939-1951 \begin{tabular}{ll|l} 
and Biochemistry & $\begin{array}{l}\text { DOI: 10.1159/000445555 } \\
\text { Published online: May 09, } 2016\end{array}$ & $\begin{array}{l}\text { ( ) 2016 The Author(s). Published by S. Karger AG, Basel } \\
\text { www.karger.com/cpb }\end{array}$ \\
\hline
\end{tabular}}

Li et al.: Periplocin Induced Apoptosis via ERK1/2-EGR1

Immunohistochemistry

Immunohistochemistry was performed to detect the levels of Mcl-1, pro-bid, pro-caspase-3, Ki67, EGR1 and p-ERK1/2 in tumor tissue. The slides of tumor tissue were deparaffinized with xylene and rehydrated through a series of ethanol concentrations. Antigens were retrieved by boiling under pressure in ethylene di-ammonium tetra acid (EDTA) buffer $(\mathrm{pH}=9.0)$ for 3 minutes. Sections were incubated with $0.3 \%$ $\mathrm{H}_{2} \mathrm{O}_{2}$ for 20 minutes and blocked with goat serum for 45 minutes followed by washing with PBS. Sections were then incubated with primary antibodies of Mcl-1, Bid, caspase-3, Ki-67, EGR1 and p-ERK1/2 (dilution 1:100) overnight at $4^{\circ} \mathrm{C}$. The next day, sections were incubated with the secondary antibody for 30 minutes at $37^{\circ} \mathrm{C}$ followed by incubation with the HRP labeled streptavidin solution for 30 minutes. PBS was used for washing after each step. After visualization of the positive antigen antibody reaction by incubation with 3 , 3-diaminobenzidine-tetrachloride (DAB) for 5 minutes, sections were counterstained with hematoxylin and evaluated by light microscopy.

\section{Statistical analysis}

SPSS13.0 software was utilized to analyze data, which was expressed as the mean \pm SEM. One-way ANOVA test was used to examine the significant differences in the experimental data between the groups. Within the two groups, data were compared using SNK-q. The threshold for significance was defined as $P<$ 0.05. All experiments were repeated three times independently.

\section{Results}

Periplocin inhibits the cell viability of gastric cancer cells

The effect of periplocin on the viability of gastric cancer cell lines SGC-7901, MGC803 and BGC-823 was determined using the MTS assay. Cells were treated with different

Fig. 1. Effect of periplocin on the viability of gastric cancer cells. (A) Gastric cancer cells (SGC-7901, MGC-803, BGC-823 and GES-1) were treated with various concentrations of periplocin $(0,50,100$, and 200 $\mathrm{ng} / \mathrm{ml}$ ) for $24 \mathrm{~h}$ and $48 \mathrm{~h}$. Cell viability was determined by MTS assays. Data are shown as the mean \pm SEM. ${ }^{*} P<0.05$ compared to the untreated cell group. (B) Cell morphology of gastric cancer cell lines treated with periplocin $(0,50,100$, and $200 \mathrm{ng} / \mathrm{ml})$.

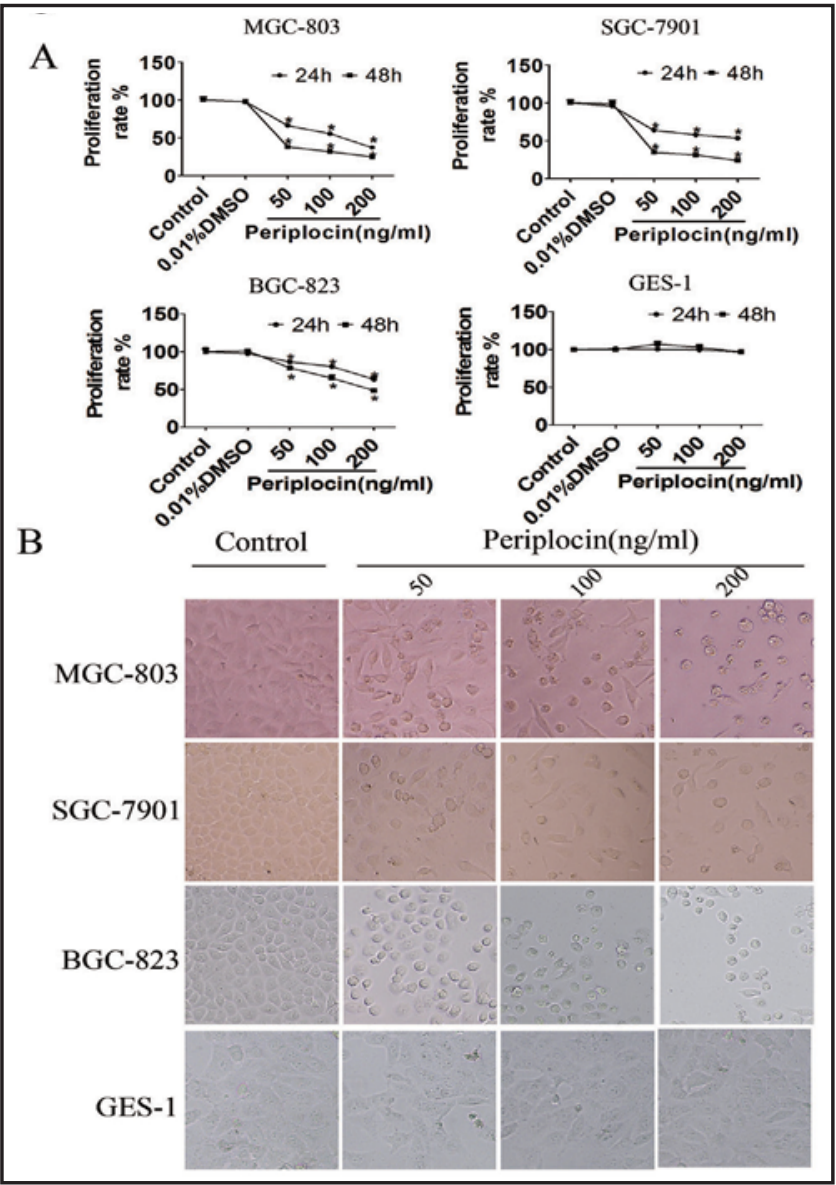




\begin{tabular}{|c|c|c|}
\hline & Cell Physiol Biochem 2016 & 38:1939-1951 \\
\hline nd Biochemistry & $\begin{array}{l}\text { DOI: 10.1159/000445555 } \\
\text { Published online: May 09, } 2016\end{array}$ & $\begin{array}{l}\text { O } 2016 \text { The Author(s). Published by S. Karger AG, Base } \\
\text { www.karger.com/cpb }\end{array}$ \\
\hline
\end{tabular}

Fig. 2. Periplocin induces apoptosis in gastric cancer cells. Flow cytometry analysis of (A) SGC-7901 cells and (B) MGC-803 cells treated with different concentrations of periplocin for $24 \mathrm{~h}$ and examined by Annexin-V/ PI double staining. The lower right quadrant indicates the percentage of early apoptotic cells and the upper right quadrant indicates the percentage of late apoptotic cells. ${ }^{*} P<0.05$ compared to the untreated cell group.

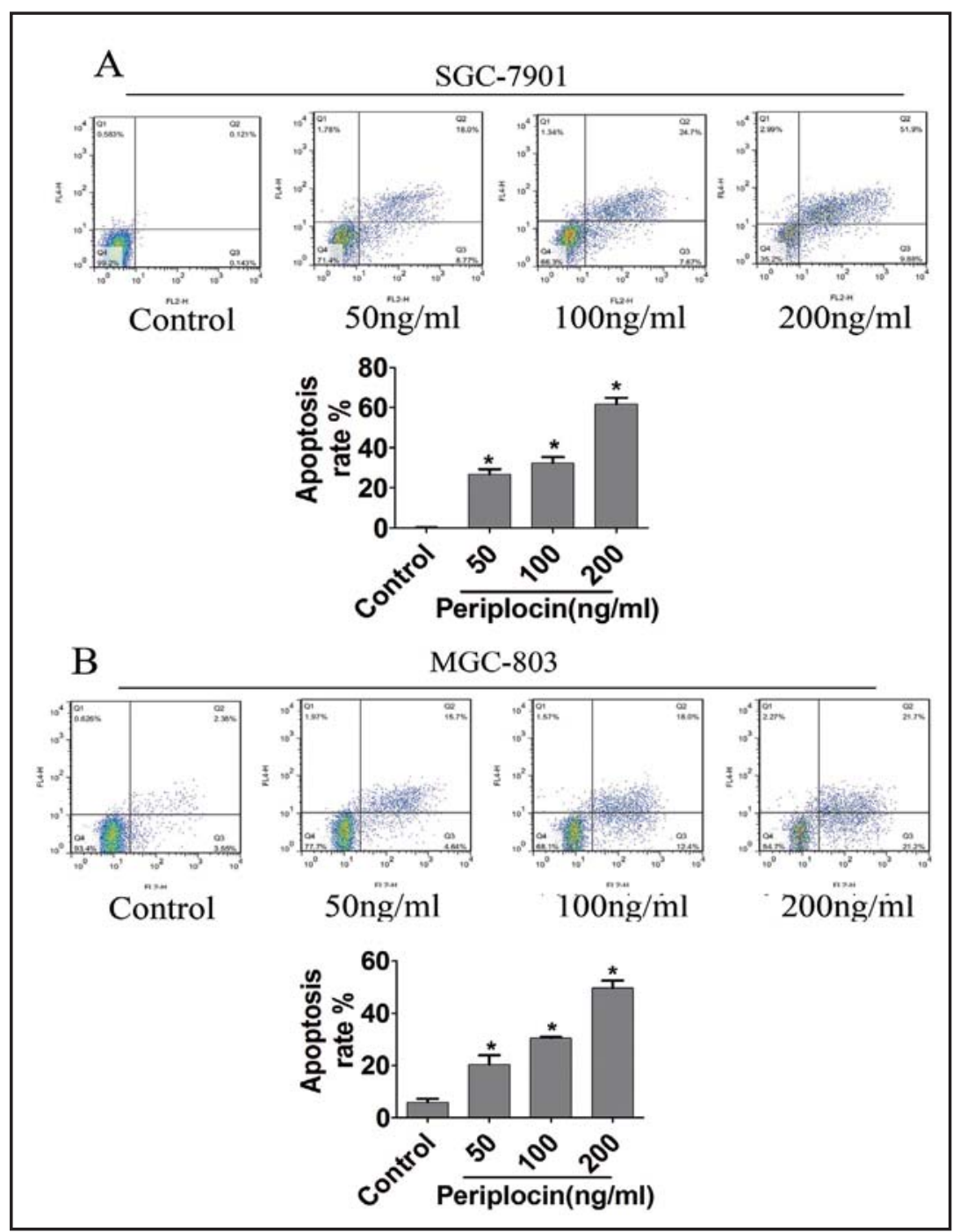

concentrations of periplocin $(0,50,100$, or $200 \mathrm{ng} / \mathrm{ml})$ for $24 \mathrm{~h}$ and $48 \mathrm{~h}$. A dose- and timedependent inhibition of the viability of gastric cancer cells was observed (Fig. 1A). After treatment with periplocin $(50,100$, or $200 \mathrm{ng} / \mathrm{ml})$ for $24 \mathrm{~h}$, the cell viability of SGC-7901 and MGC-803 cells ranged from $63.94 \%$ to $53.39 \%(P<0.05)$ and $66.43 \%$ to $37.06 \%(P<0.05)$, respectively. A similar effect was also observed in BGC-823 cells. However, periplocin had no effect on GES-1 cells $(P>0.05)$. We also detected dramatically altered cell morphology in cells treated with periplocin (Fig. 1B). The shape of SGC-7901, MGC-803 and BGC-823 cells treated with periplocin appeared round compared to untreated cells. However, no change was observed in GES-1 cells.

Treatment of gastric cancer cells with periplocin induces apoptosis

To investigate whether periplocin induced apoptosis, SGC-7901 and MGC-803 cells were treated with varying doses of periplocin for $24 \mathrm{~h}$, and the number of apoptotic cells was determined by flow cytometry using Annexin V-FITC staining. The lower right and upper right quadrants of the histogram represent early and late stage apoptotic cells, respectively (Fig. 2). Treatment of SGC-7901 and MGC-803 cells with periplocin for $24 \mathrm{~h}$ resulted in induction of apoptotic cell death in both cell lines. Apoptotic cells among SGC-7901 and MGC-803 cells after periplocin (50,100, or $200 \mathrm{ng} / \mathrm{ml}$ ) treatment ranged from $26.77 \%$ to $61.78 \%(P<0.05)$ and $20.34 \%$ to $42.90 \%(P<0.05)$, respectively, compared to only $0.27 \%$ and $5.93 \%$ in untreated control cells. 
Fig. 3. TUNEL assay of gastric cancer cells treated with periplocin. TUNEL staining of (A) SGC-7901 cells and (B) MGC-803 cells treated with different concentrations of periplocin for $24 \mathrm{~h}$. The number of apoptotic cells was increased in the periplocin treated group. ${ }^{*} P<0.05$ compared to the untreated cell group.

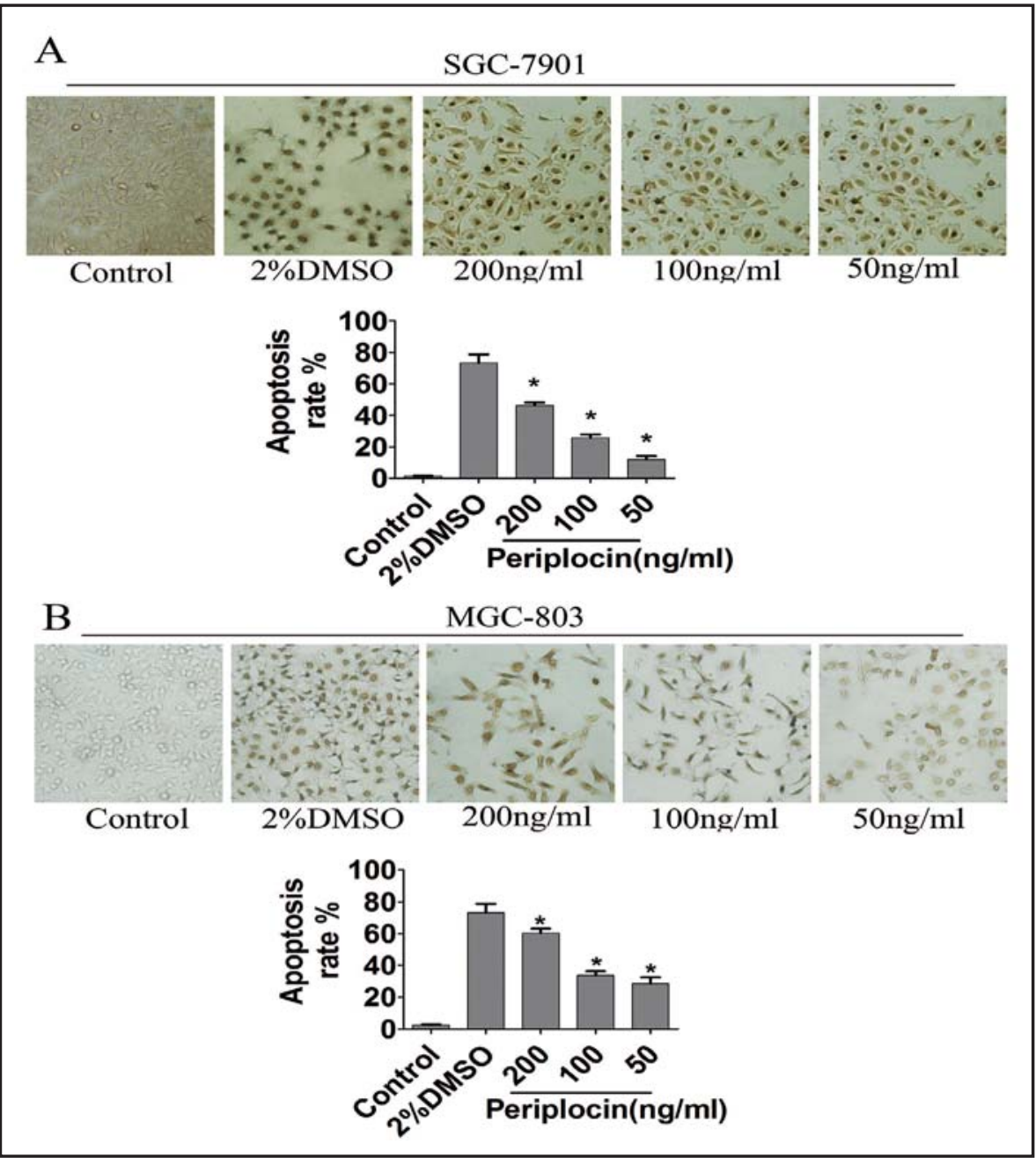

TUNEL assays confirmed that treatment of SGC-7901 and MGC-803 cells with periplocin for $24 \mathrm{~h}$ resulted in a significant increase in apoptotic cells (Fig. 3).

Periplocin induces changes in the expression of apoptosis-related proteins

To investigate the potential mechanism involved in periplocin-induced apoptosis, we determined the expression of various apoptosis-related molecules by western blot analysis (Fig. 4). Treatment of SGC-7901 and MGC-803 cells with periplocin $(0,50,100$, or $200 \mu \mathrm{g} / \mathrm{ml})$ for $24 \mathrm{~h}$ resulted in a dose-dependent decrease in the expression of anti-apoptotic protein, Mcl-1, while pro-Bid, decreased with increasing doses of periplocin. In addition, the results revealed a gradual increase of cleaved caspase- 3 in a dose-dependent manner.

\section{Periplocin was identified as a differential expressed gene by microarray}

Next, we performed a differential gene expression analysis between untreated and 100 $\mathrm{ng} / \mathrm{ml}$ periplocin treated SGC-7901 cells. There were many differentially expressed genes identified by the Affymetrix Human Genome U133 Plus 2.0 Array. The genes that exhibit the most significant fold changes and are related to cancer growth are shown in the Table 1 . We verified these results by real-time PCR (Fig. 5A). The results of pathway analysis performed with differentially expressed genes showed that several signals were related to periplocin inhibited proliferation of gastric cancer cells, such as RhoA signaling, GADD45 signaling and p53 signaling (Fig. 8).

Many studies have reported that upregulation of EGR1 expression can inhibit proliferation of breast cancer, non-small cell lung carcinoma and esophageal squamous cell 


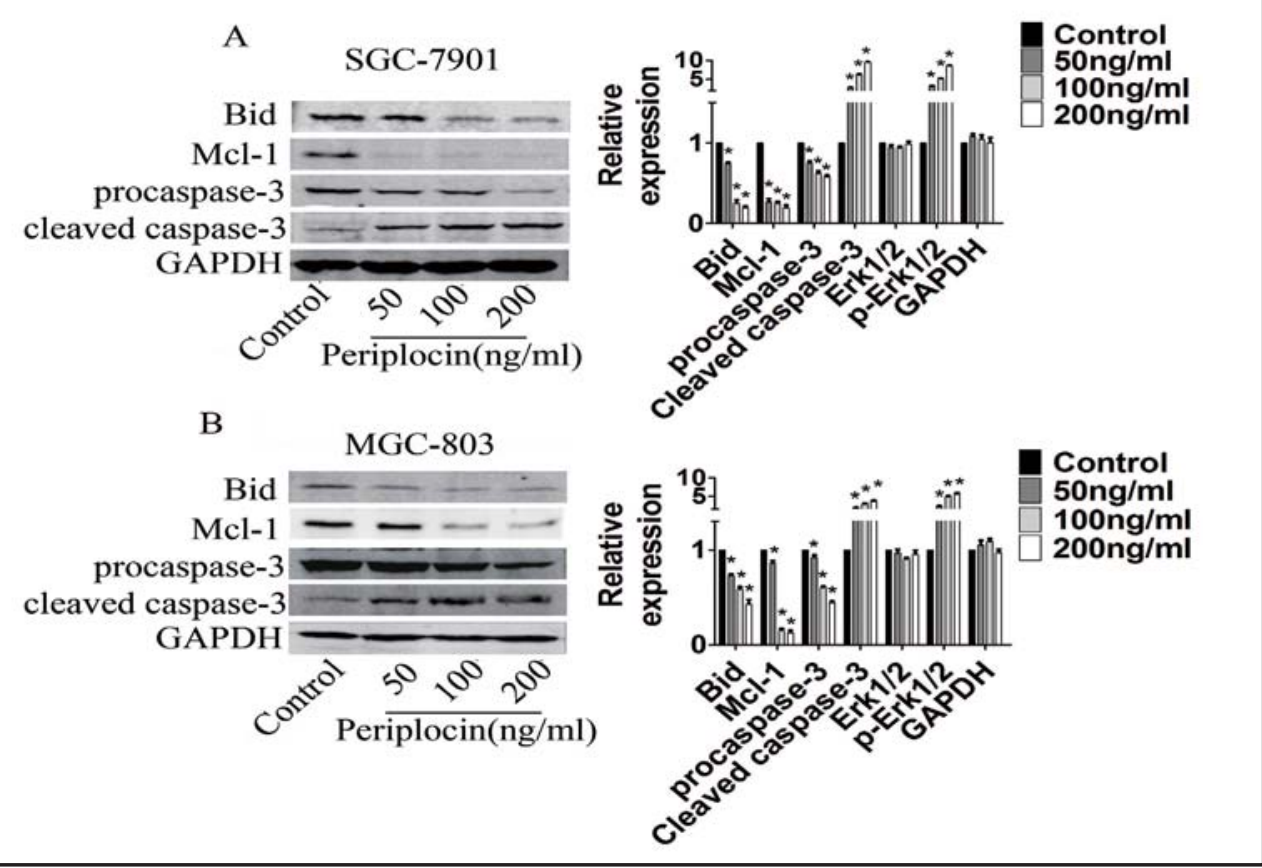

Fig. 4. Periplocin induces changes in the expression of apoptosis-related proteins. Western blot analysis of pro-bid, Mcl-1, cleaved caspase-3 and p-ERK1/2 protein levels in (A) SGC-7901 cells and (B) MGC-803 cells treated with periplocin for $24 \mathrm{~h} .{ }^{*} P<0.05$ compared to the untreated cell group.

Table 1. Differential gene expression in SGC-7901 cells treated with periplocin compared to untreated cells

\begin{tabular}{|c|c|c|}
\hline Gene & $\begin{array}{l}\text { Fold-Change } \\
\text { (t vs. c) }\end{array}$ & Description \\
\hline ID2 & 26.4446 & inhibitor of DNA binding 2, dominant negative helix-loop-helix \\
\hline LINC00273 & 13.1932 & long intergenic non-protein coding RNA 273 \\
\hline RN45S & 11.0344 & $45 \mathrm{~S}$ pre-ribosomal RNA \\
\hline TMEM27 & 9.54151 & transmembrane protein 27 \\
\hline RN45S & 9.18391 & $45 \mathrm{~S}$ pre-ribosomal RNA \\
\hline EGR1 & 7.65261 & early growth response 1 \\
\hline PTGS2 & 6.66579 & prostaglandin-endoperoxide synthase 2 \\
\hline ID2 & 6.62147 & inhibitor of DNA binding 2, dominant negative helix-loop-helix protein \\
\hline UGCG & 6.30983 & UDP-glucose ceramide glucosyltransferase \\
\hline NR4A3 & 6.173 & nuclear receptor subfamily 4 , group A, member 3 \\
\hline AREG & 5.56132 & amphiregulin \\
\hline DKK1 & 5.52635 & dickkopf 1 homolog (Xenopus laevis) \\
\hline SRGAP1 & 5.525 & SLIT-ROBO Rho GTPase activating protein 1 \\
\hline ARL5B & 5.15782 & ADP-ribosylation factor-like $5 \mathrm{~B}$ \\
\hline GEM & 5.13883 & GTP binding protein overexpressed in skeletal muscle \\
\hline DUSP4 & 5.1209 & dual specificity phosphatase 4 \\
\hline DUSP4 & 5.06099 & dual specificity phosphatase 4 \\
\hline SERPINB5 & -9.56029 & serpin peptidase inhibitor, clade B (ovalbumin), member 5 \\
\hline KCTD18 & -6.99119 & potassium channel tetramerisation domain containing 18 \\
\hline DGKA & -5.9721 & diacylglycerol kinase, alpha $80 \mathrm{kDa}$ \\
\hline SORBS2 & -5.82356 & sorbin and $\mathrm{SH} 3$ domain containing 2 \\
\hline MEGF6 & -5.60072 & multiple EGF-like-domains 6 \\
\hline ASB9 & -5.46465 & ankyrin repeat and SOCS box containing 9 \\
\hline CHD9 & -5.42373 & chromodomain helicase DNA binding protein 9 \\
\hline LOC728431 & -5.25579 & uncharacterized LOC728431 \\
\hline NUCKS1 & -5.05523 & nuclear casein kinase and cyclin-dependent kinase substrate 1 \\
\hline
\end{tabular}



Cellular Physiology Cell Physiol Biochem 2016;38:1939-1951 \begin{tabular}{c|c|c|c|}
\hline DOI: 10.1159/000445555 & 2016 The Author(s). Published by S. Karger AG, Basel
\end{tabular}
Li et al.: Periplocin Induced Apoptosis via ERK1/2-EGR1

Fig. 5. Periplocin activates p-ERK1/2/ EGR1 and suppresses gastric cancer cell viability via EGR1. (A) Real-time PCR analysis of EGR1, H19 and SerpinB5 mRNA levels in SGC-7901 cells treated with periplocin for 24 h. (B) Western blot analysis of EGR1 and p-ERK1/2 protein levels in SGC7901 and MGC-803 cells treated with periplocin for $24 \mathrm{~h}$. (C) RT-PCR analysis of EGR1 mRNA levels in SGC-7901 and MGC803 cells transfected with EGR1 siRNA. (D) MTS assay of SGC7901 and MGC-803 cells transfected with EGR1 siRNA or negative control siRNA and treated with periplocin.

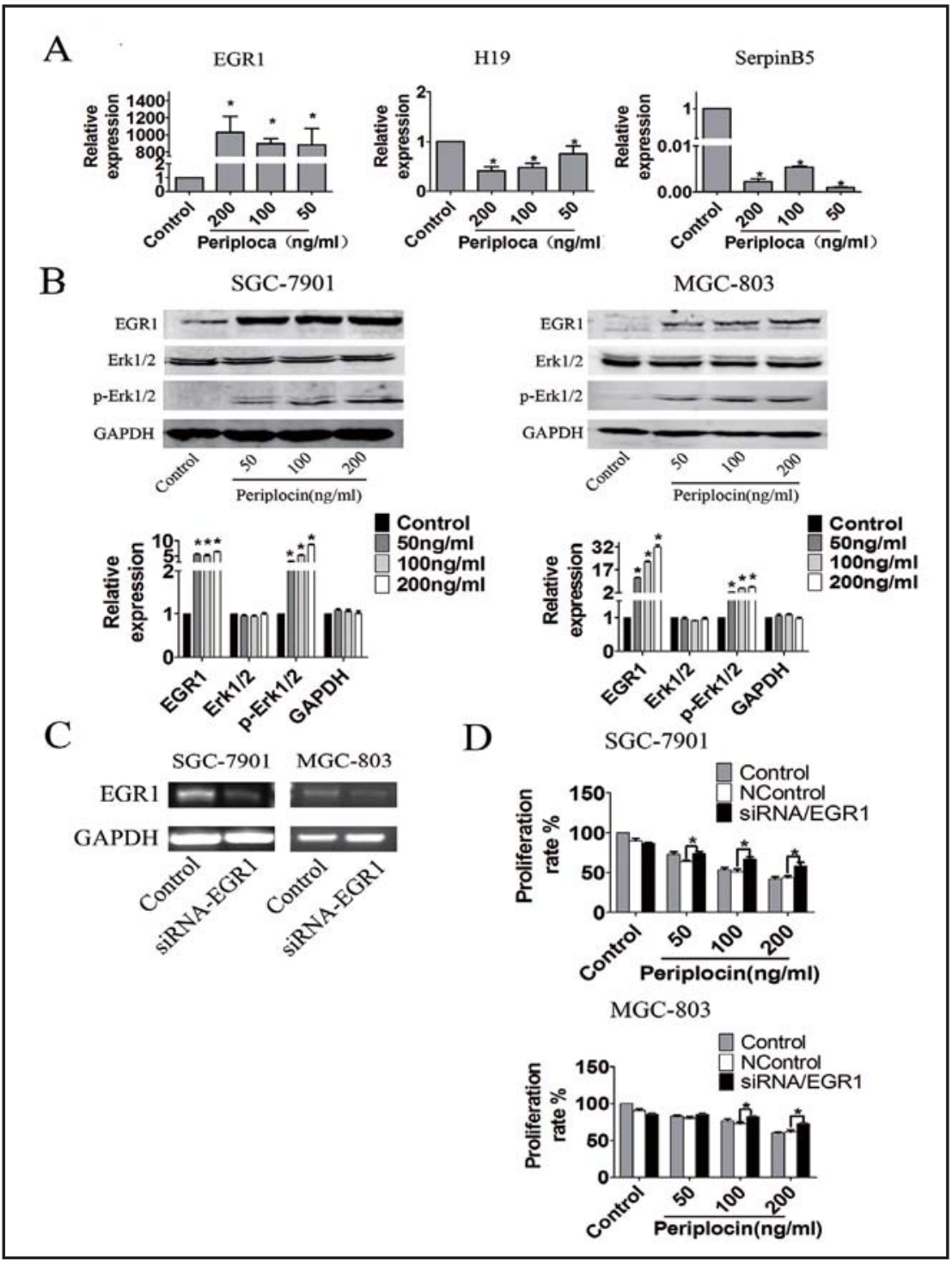

carcinoma cells [9-11]. Our microarray results also showed increased expression of EGR1 in SGC-7901 cells treated with periplocin. Therefore, we examined the role of EGR1 in greater detail to study the mechanism of inhibition of cell viability by periplocin in gastric cancer cells.

\section{Periplocin upregulated the expression of EGR1 and ERK1/2}

We evaluated the expression of EGR1 protein in SGC-7901 and MGC-803 cells treated with periplocin by western blot and obtained similar results as the microarray findings (Fig. 5B). ERK1/2 is an upstream regulators of EGR1 [12], so we also examined its expression and activation (phosphorylation) status. The western blot results showed that periplocin also upregulated the expression of p-ERK1/2 (Fig. 5B). Together, these results suggest that periplocin may inhibit the cell viability of gastric cancer cells by the ERK1/2-EGR1 pathway.

Knockdown of EGR1 could rescue the cell viability of gastric cancer cells treated with periplocin

To more closely examine the role of EGR1 in periplocin suppression of gastric cancer cell viability, we performed knockdown of EGR1 using siRNA transfection. Effective downregulation of EGR1 in cell lines was verified using RT-PCR (Fig. 5C). MTS assay analysis 

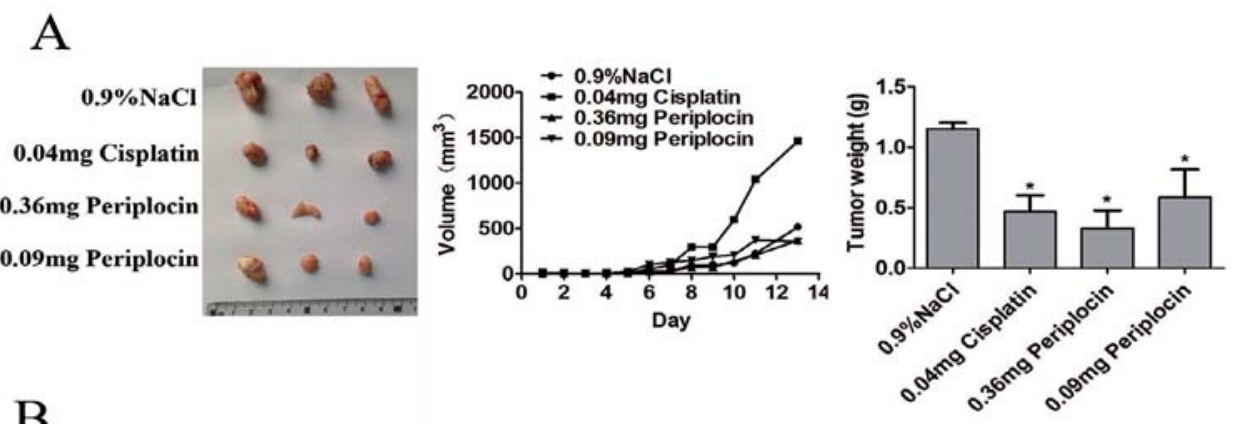

B

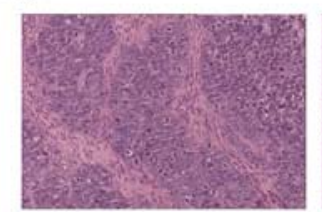

$0.9 \% \mathrm{NaCl}$

C

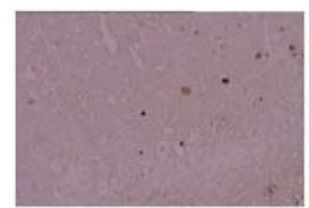

$0.9 \% \mathrm{NaCl}$

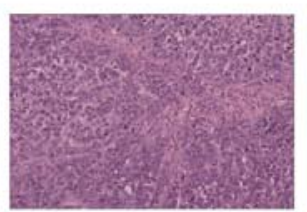

$0.09 \mathrm{mg}$ Cisplatin

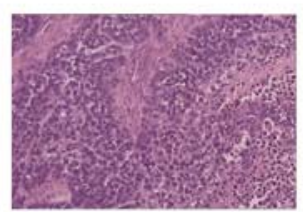

0.36mg Periplocin

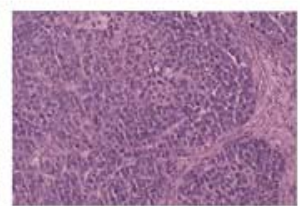

0.09mg Periplocin

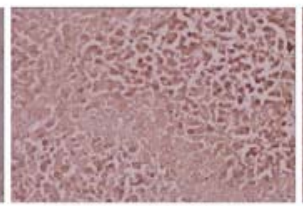

0.09mg Cisplatin

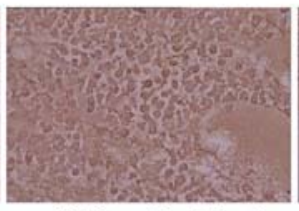

0.36mg Periplocin

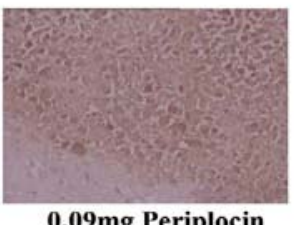

0.09mg Periplocin

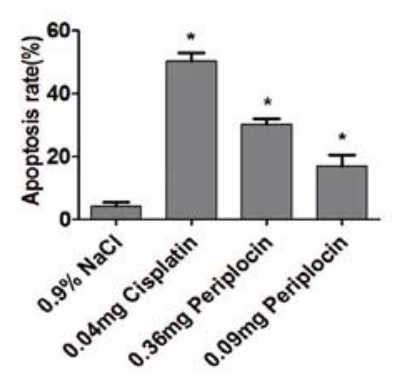

Fig. 6. Effects of periplocin on gastric tumors in nude mice. (A) Representative images from each treatment group are presented. The sizes of the tumor xenografts were recorded daily in each treatment group. Upon termination of the experiment, wet tumor weight was measured. Significant inhibition in tumor xenograft growth and tumor weight was recorded in the periplocin-treated mice. ${ }^{*} P<0.05$ compared to the control group. (B) Micrographs of hematoxylin/eosin-stained sections of tumor tissues. (C) Comparison of the apoptosis levels in the four treatment groups by TUNEL staining. The high dose group and positive group had significantly increased cellular apoptotic levels in the tumor tissues compared to controls. The apoptotic level of the low dose group also increased compared to controls.

demonstrated that the viability of cells knocked down for EGR1 expression was increased compared to cells transfected with control siRNA and treated with periplocin (Fig. 5D). Together these data suggest that periplocin may inhibit cell viability of gastric cancer cells via the ERK1/2-EGR1 pathway.

Effects of periplocin on gastric tumor formation in nude mice

To further explore the effects of periplocin in gastric cancer cells, we examined the inhibition of periplocin on tumor formation using SGC-7901 cells in BALB/c nude mice in vivo. Mice treated with periplocin did not exhibit impaired movement or any other visible sign of 


\section{Cellular Physiology Cell Physiol Biochem 2016;38:1939-1951 \begin{tabular}{l|l|l}
\cline { 2 - 2 } aOI: 10.1159/000445555 & $\begin{array}{l}\text { O 2016 The Author(s). Published by S. Karger AG, Basel } \\
\text { www.karger.com/cpb }\end{array}$ \\
\hline
\end{tabular}}

Li et al.: Periplocin Induced Apoptosis via ERK1/2-EGR1

Fig. 7. Immunohistochemistry of apoptosis-related proteins in vivo. Immunohistochemical staining for Mcl-1, Bid, caspase-3, Ki-67, EGR1 and p-ERK1/2 in tumor tissues. Expression levels of Mcl-1, pro-Bid, pro-caspase-3 and Ki-67 were decreased and expression levels of EGR1 and p-ERK1/2 were increased in periplocin-treated groups compared to controls.

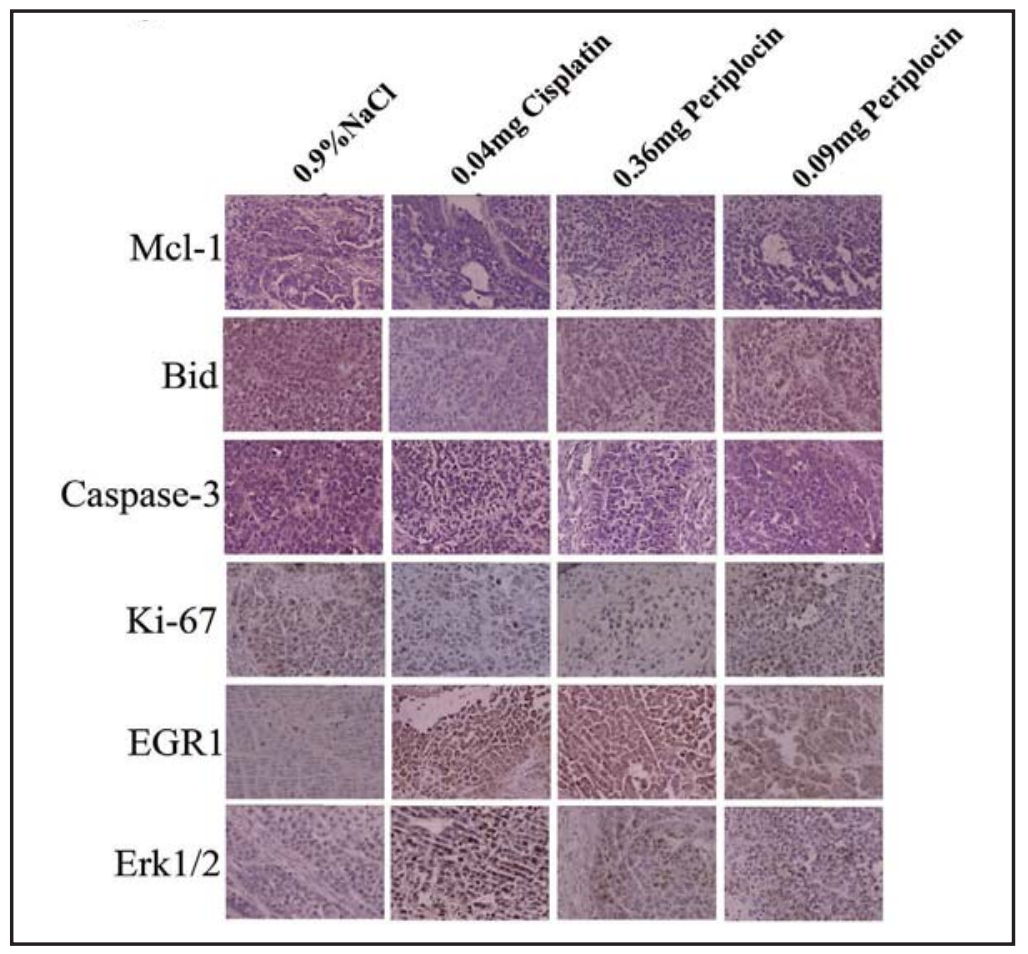

physical toxicity. After 14 days, tumors were harvested and weighed (Fig. 6A, B). The tumor volumes of all drug-treated groups (Groups II, III, and IV) were significantly lower than the control group (Group I). The tumor volume in the group treated with a high concentration of periplocin (Group III) was similar to the cisplatin positive control (Group II).

To examine whether periplocin can lead to apoptosis of SGC-7901 cells in vivo, we performed TUNEL assays in tumor tissues (Fig. 6C). The results showed significantly higher numbers of apoptotic cells in the groups treated by cisplatin and periplocin compared to the control group.

To investigate the potential mechanism by which periplocin induces apoptosis in vivo, we performed immunohistochemistry on apoptosis-related molecules in tumor tissues. As shown in Fig. 7, the expression levels of Mcl-1, pro-caspase-3, pro-Bid and Ki-67 were decreased in all treatment groups compared with the control group. Notably, the expression levels of EGR1 and p-ERK1/2 were also increased in all treatment groups compared with the control group.

\section{Discussion}

Gastric cancer is associated with malignant tumors and with high incidence and mortality [13]. Identification of novel treatments for gastric cancer is required given the low sensitivity and high toxicity of chemotherapeutic drugs. Periplocin is a monomer compound obtained from cortex periplocae by butanol extraction. Previous studies have demonstrated that periplocin exhibits a potent inhibitory effect on the growth of several tumor cell lines $[8,14]$. However, its effects on human gastric carcinoma cells have not yet been examined. In this study, we verified the effect of periplocin on gastric cancer cells using both in vitro and in vivo models.

Cancer is associated not only with abnormal proliferation and differentiation of cells but also with abnormal apoptosis $[15,16]$. Apoptosis or programmed cell death is a process that results in the controlled elimination of unhealthy or damaged cells [17]. Many studies have reported that apoptosis contributes to the inhibition of tumor cell proliferation in malignant 


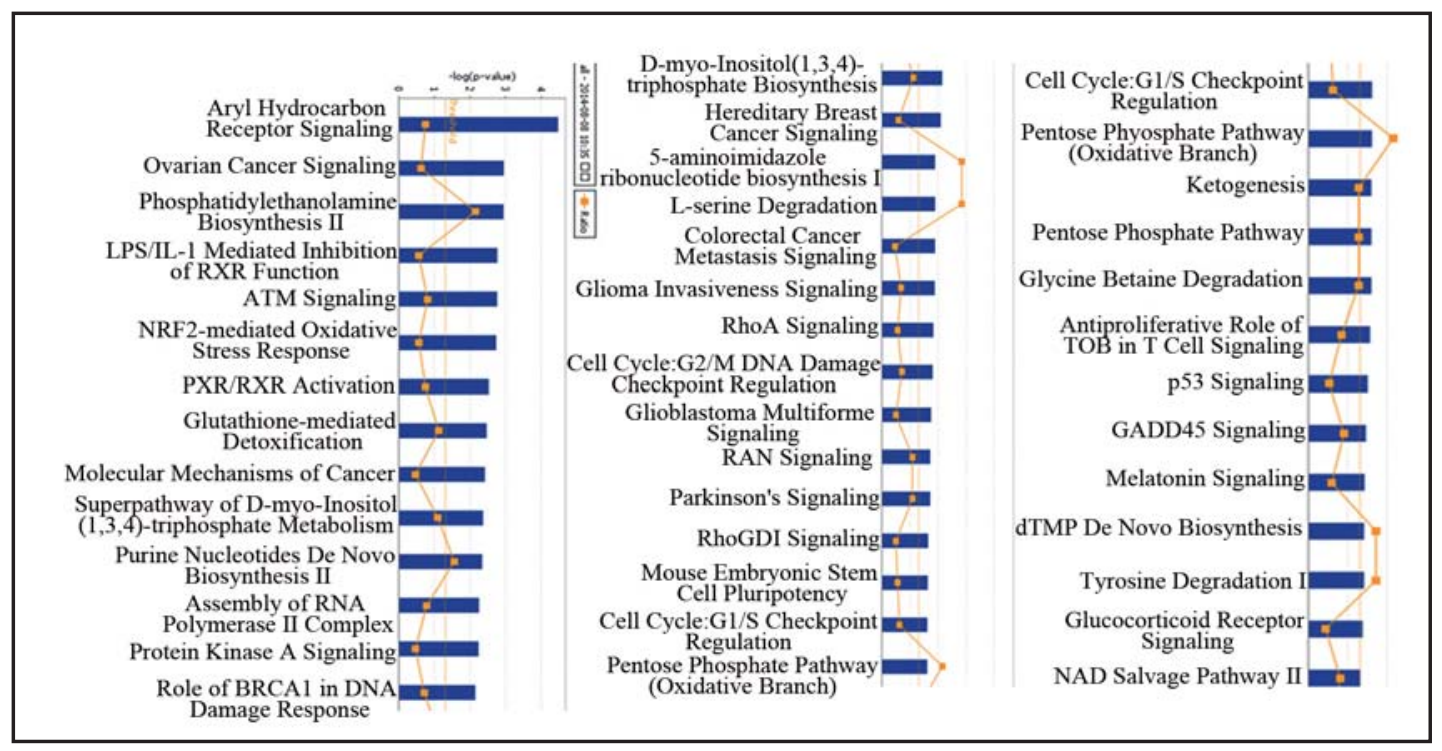

Fig. 8. The results of performing the pathway analysis with differentially expressed genes.

tumors [18-20]. We examined the effect of peroplocin on gastric cancer cells by MTS assays and found that after treatment with periplocin for $24 \mathrm{~h}$ and $48 \mathrm{~h}$, the viability of several gastric cancer cell lines was significantly reduced. In addition, flow cytometry and TUNEL assays confirmed a dose-dependent increase in apoptosis in SGC-7901 and MGC-803 gastric cancer cells upon treatment with periplocin. Apoptosis is a complex cellular mechanism governed by several signaling molecules $[21,22]$. The Bcl-2 family mediates a major apoptotic signal transduction cascade and includes anti-apoptotic and pro-apoptotic proteins [23, 24]. We found that induction of apoptosis in SGC-7901 and MGC-803 cells by periplocin was associated with a decrease in the levels of the anti-apoptotic protein Mcl-1 and non-activated Bid, a pro-apoptotic protein. In addition, the expression level of cleaved caspase-3 was increased by periplocin. Caspases are a group of cysteine-containing proteolytic enzymes that play a critical role in mediating apoptosis $[25,26]$. Caspase-3 is a key enzyme that is activated by cleavage at the start of apoptosis and is one of the crucial downstream factors in executing the apoptotic program $[27,28]$. Our in vivo immunohistochemistry and TUNEL assay results were consistent with these findings. Together, these data indicate that the inhibitory effects of periplocin on gastric cancer cells are mediated by induction of apoptosis.

The microarray results showed that several genes and pathways were modified upon treatment of gastric cancer cells with periplocin. In particular, the expression level of EGR1 was increased significantly. EGR1 is activated by periplocin in gastric cancer cells in vitro and in vivo, and knockdown of EGR1 can rescue gastric cancer cells treated with periplocin. The EGR1 gene is a member of the immediate early gene family encoding a zinc finger transcriptional factor [29] and is an important regulator of cell growth, differentiation and survival that is known to play a role in carcinogenesis and cancer progression [30]. Given that EGR1 is known to be upregulated via the activation of ERK1/2 [31], we also analyzed blots for increases in ERK1/2 phosphorylation. Our results showed that increased ERK1/2 phosphorylation appeared to correlate with the increases seen in EGR1 levels. Our in vivo immunohistochemistry results were consistent with these findings. Together these data indicate that periplocin might inhibit the cell viability of gastric cancer cells via the ERK1/2EGR1 pathway.

In conclusion, our results show that periplocin can inhibit the proliferation of gastric cancer cells by inducing apoptosis in vitro and in vivo and that the ERK1/2-EGR1 pathway may mediate the cellular effects of periplocin. These findings provide an experimental basis for using periplocin as a chemotherapeutic drug against gastric cancer cells, thereby facilitating the development of new anticancer agents. 


\section{Cellular Physiology Cell Physiol Biochem 2016;38:1939-1951 \begin{tabular}{ll|l} 
DOI: 10.1159/000445555 & $\begin{array}{l}\text { ( ) 2016 The Author(s). Published by S. Karger AG, Basel } \\
\text { www.karger.com/cpb }\end{array}$
\end{tabular}}

Li et al.: Periplocin Induced Apoptosis via ERK1/2-EGR1

\section{Acknowledgments}

This study was supported by the National Natural Science Foundation of China (Grant No. 81502032, 81173611), Hebei Province Science and Technology Plan Project (Grant No.H2015206376) and Financial department of Hebei Province (Grant No.20142065).

\section{Disclosure Statement}

The authors have declared no conflicts of interest.

\section{References}

1 Crew KD, Neugut AI: Epidemiology of gastric cancer. World J Gastroenterol 2006;12:354-362.

-2 Jayavelu ND, Bar NS: Metabolomic studies of human gastric cancer: Review. World J Gastroenterol 2014;20:8092-8101.

-3 Sant M, Allemani C, Santaquilani M, Knijn A, Marchesi F, Capocaccia R, Group EW: EUROCARE-4. Survival of cancer patients diagnosed in 1995-1999. Results and commentary. Eur J Cancer 2009;45:931-991.

-4 Mizoshita T, Kataoka H, Kubota E, Okamoto Y, Shimura T, Mori Y, Wada T, Ogasawara N, Sasaki M, Kamiya T, Joh T: Gastric phenotype signet-ring cell carcinoma of the stomach with multiple bone metastases effectively treated with sequential methotrexate and 5-fluorouracil. Int J Clin Oncol 2008;13:373-376.

5 Guo H, Mao H, Pan G, Zhang H, Fan G, Li W, Zhou K, Zhu Y, Yanagihara N, Gao X: Antagonism of Cortex Periplocae extract-induced catecholamines secretion by Panax notoginseng saponins in cultured bovine adrenal medullary cells by drug combinations. J Ethnopharmacol 2013;147:447-455.

-6 Itokawa H XJ, Takeya K, Watanabe K, Shoji J: Studies on chemical constituents of antitumor fraction from Periploca sepium. II. Structures of new pregnane glycosides, periplocosides A, B and C. Chem Pharm Bull (Tokyo) 1988;36:982-987.

7 Zhao L, Shan B, Du Y, Wang M, Liu L, Ren FZ: Periplocin from Cortex periplocae inhibits cell growth and down-regulates survivin and c-myc expression in colon cancer in vitro and in vivo via $\beta$-catenin/TCF signaling. Oncol Rep 2010;24:375-383.

8 Lu ZJ ZY, Song Q, Qin Z, Zhang H, Zhou YJ, Gou LT, Yang JL, Luo F: Periplocin Inhibits Growth of Lung Cancer in vitro and in vivo by Blocking AKT/ERK Signaling Pathways. Cell Physiol Biochem 2010;26:609-618.

-9 Li ZL, Liang S, Wang ZC, Li YB, Guo CX, Fang F, Gong SL, Lin CH: Expression of Smac induced by the Egr1 promoter enhances the radiosensitivity of breast cancer cells. Cancer Gene Ther 2014;21:142-149.

10 Dong Q Zhang J, Hendricks DT, Zhao X: GRObeta and its downstream effector EGR1 regulate cisplatininduced apoptosis in WHCO1 cells. Oncol Rep 2011;25:1031-1037.

-11 Zhang H, Chen X, Wang J, Guang W, Han W, Zhang H, Tan X, Gu Y: EGR1 decreases the malignancy of human non-small cell lung carcinoma by regulating KRT18 expression. Sci Rep 2014;4:5416.

12 Keates S, Keates AC, Nath S, Peek RM Jr, Kelly CP: Transactivation of the epidermal growth factor receptor by cag+ Helicobacter pylori induces upregulation of the early growth response gene Egr-1 in gastric epithelial cells. Gut 2005;54:1363-1369.

13 Anderson WF, Camargo MC, Fraumeni JF Jr, Correa P, Rosenberg PS, Rabkin CS: Age-specific trends in incidence of noncardia gastric cancer in US adults. JAMA 2010;303:1723-1728.

14 Du YY LX, Shan BE: Periplocin extracted from cortex periplocae induces apoptosis of SW480 cells through inhibiting the Wnt/beta-catenin signaling pathway. Ai Zheng 2009;28:456-460.

15 Cappellari AR, Pillat MM, Souza HD, Dietrich F, Oliveira FH, Figueiro F, Abujamra AL, Roesler R, Lecka J, Sevigny J, Battastini AM, Ulrich H: Ecto-5'-Nucleotidase Overexpression Reduces Tumor Growth in a Xenograph Medulloblastoma Model. PLoS One 2015;10:e0140996.

-16 Acikgoz E, Guven U, Duzagac F, Uslu R, Kara M, Soner BC, Oktem G: Enhanced G2/M Arrest, Caspase Related Apoptosis and Reduced E-Cadherin Dependent Intercellular Adhesion by Trabectedin in Prostate Cancer Stem Cells. PLoS One 2015;10:e0141090.

17 Sugata M, Lin CY, Shih YC: Anti-Inflammatory and Anticancer Activities of Taiwanese Purple-Fleshed Sweet Potatoes (Ipomoea batatas L. Lam) Extracts. Biomed Res Int 2015;2015:768093. 


\section{Cellular Physiology Cell Physiol Biochem 2016;38:1939-1951 \begin{tabular}{ll|l} 
DOI: 10.1159/000445555 & $\begin{array}{l}\text { ( ) 2016 The Author(s). Published by S. Karger AG, Basel } \\
\text { www.karger.com/cpb }\end{array}$
\end{tabular}}

Li et al.: Periplocin Induced Apoptosis via ERK1/2-EGR1

18 Zhao X, Yang W, Pei F, Ma W, Wang Y: Downregulation of matrix metalloproteinases contributes to the inhibition of cell migration and invasion in HepG2 cells by sodium valproate. Oncol Lett 2015;10:531-535.

19 Li Y, Liu D, Zhou Y, Li Y, Xie J, Lee RJ, Cai Y, Teng L: Silencing of Survivin Expression Leads to Reduced Proliferation and Cell Cycle Arrest in Cancer Cells. J Cancer 2015;6:1187-1194.

20 Li X, Li H, Zhang R, Liu J, Liu J: MicroRNA-449a inhibits proliferation and induces apoptosis by directly repressing E2F3 in gastric cancer. Cell Physiol Biochem 2015;35:2033-2042.

-21 Farias CF, Massaoka MH, Girola N, Azevedo RA, Ferreira AK, Jorge SD, Tavares LC, Figueiredo CR, Travassos LR: Benzofuroxan derivatives $\mathrm{N}-\mathrm{Br}$ and $\mathrm{N}-\mathrm{I}$ induce intrinsic apoptosis in melanoma cells by regulating AKT/BIM signaling and display anti metastatic activity in vivo. BMC Cancer 2015;15:807.

22 Sun H, Gao Y, Lu K, Zhao G, Li X, Li Z, Chang H: Overexpression of Klotho suppresses liver cancer progression and induces cell apoptosis by negatively regulating wnt/beta-catenin signaling pathway. World J Surg Oncol 2015;13:307.

-23 Schoneich C, Dremina E, Galeva N, Sharov V: Apoptosis in differentiating C2C12 muscle cells selectively targets Bcl-2-deficient myotubes. Apoptosis 2014;19:42-57.

24 Zhu P, Zhang J, Zhu J, Shi J, Zhu Q, Gao Y: MiR-429 Induces Gastric Carcinoma Cell Apoptosis Through Bcl-2. Cell Physiol Biochem 2015;37:1572-1580.

25 Frejlich E R-RJ, Janiszewski K, Salomon L, Kotulski K, Pelzer O, Grzebieniak Z, Tarnawa R, Kielan W: Caspases and Their Role in Gastric Cancer. Adv Clin Exp Med 2013;22:593-602.

26 Yi CH, Yuan J: The Jekyll and Hyde functions of caspases. Dev Cell 2009;16:21-34.

27 Zhang R, Piao MJ, Kim KC, Kim AD, Choi JY, Choi J, Hyun JW: Endoplasmic reticulum stress signaling is involved in silver nanoparticles-induced apoptosis. Int J Biochem Cell Biol 2012;44:224-232.

28 Seo TB, Kim TW, Shin MS, Ji ES, Cho HS, Lee JM, Kim TW, Kim CJ: Aerobic exercise alleviates ischemiainduced memory impairment by enhancing cell proliferation and suppressing neuronal apoptosis in hippocampus. Int Neurourol J 2014;18:187-197.

29 Gashler A SV: Early growth response protein 1 (Egr-1): prototype of a zinc-finger family of transcription factors. Proq Nucleic Acid Res Mol Biol 1995;50:191-224.

-30 Thiel G, Cibelli G: Regulation of life and death by the zinc finger transcription factor Egr-1. J Cell Physiol 2002;193:287-292.

-31 Al-Sarraj A TG: Substance P induced biosynthesis of the zinc finger transcription factor Egr-1 in human glioma cells requires activation of the epidermal growth factor receptor and of extracellular signalregulated protein kinase. Neuroscience Letters 2002;332:111-114. 
In the original article by Li et al. entitled "Periplocin Extracted from Cortex Periplocae Induced Apoptosis of Gastric Cancer Cells via the ERK1/2-EGR1 Pathway3" [Cell Physiol Biochem 2016;38:1939-1951 (DOI: 10.1159/000445555)] there is an error in Figures 3 and 4 . The correct figures and the legends are reproduced correctly here.

The authors terribly apologized for this carelessness.

Fig. 3. TUNEL assay of gastric cancer cells treated with periplocin. TUNEL staining of (A) SGC7901 cells and (B) MGC-803 cells treated with different concentrations of periplocin for $24 \mathrm{~h}$. The number of apoptotic cells was increased in the periplocin treated group. ${ }^{*} P<0.05$ compared to the untreated cell group.

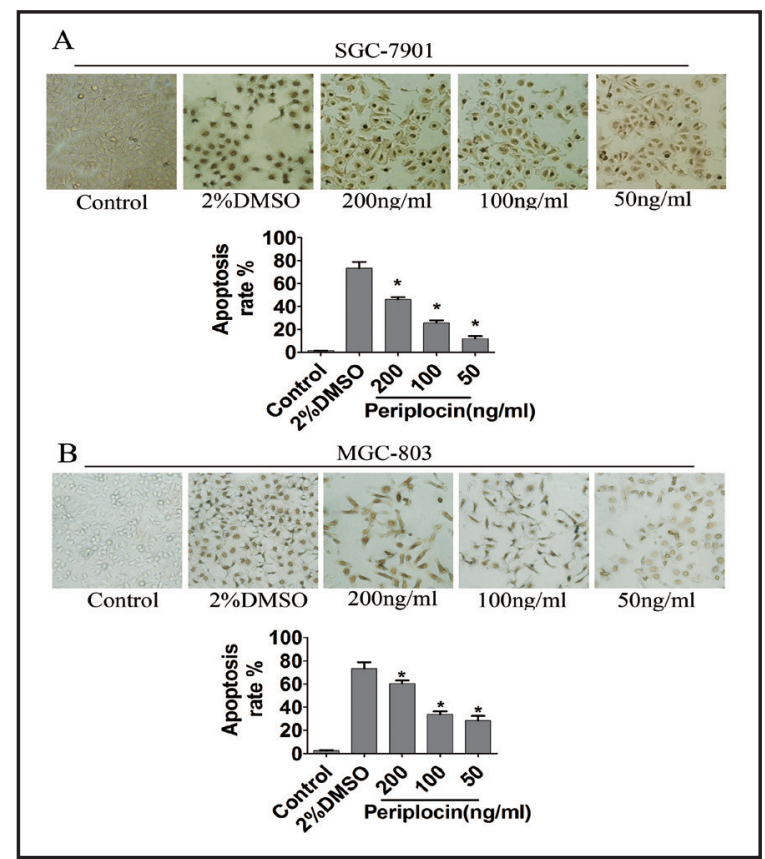

Fig. 4. Periplocin induces changes in the expression of apoptosis-related proteins. Western blot analysis of probid, Mcl-1, cleaved caspase-3 and $\mathrm{p}$-ERK1/2 protein levels in (A) SGC-7901 cells and (B) MGC-803 cells treated with periplocin for 24 h. ${ }^{*} P<0.05$ compared to the untreated cell group.

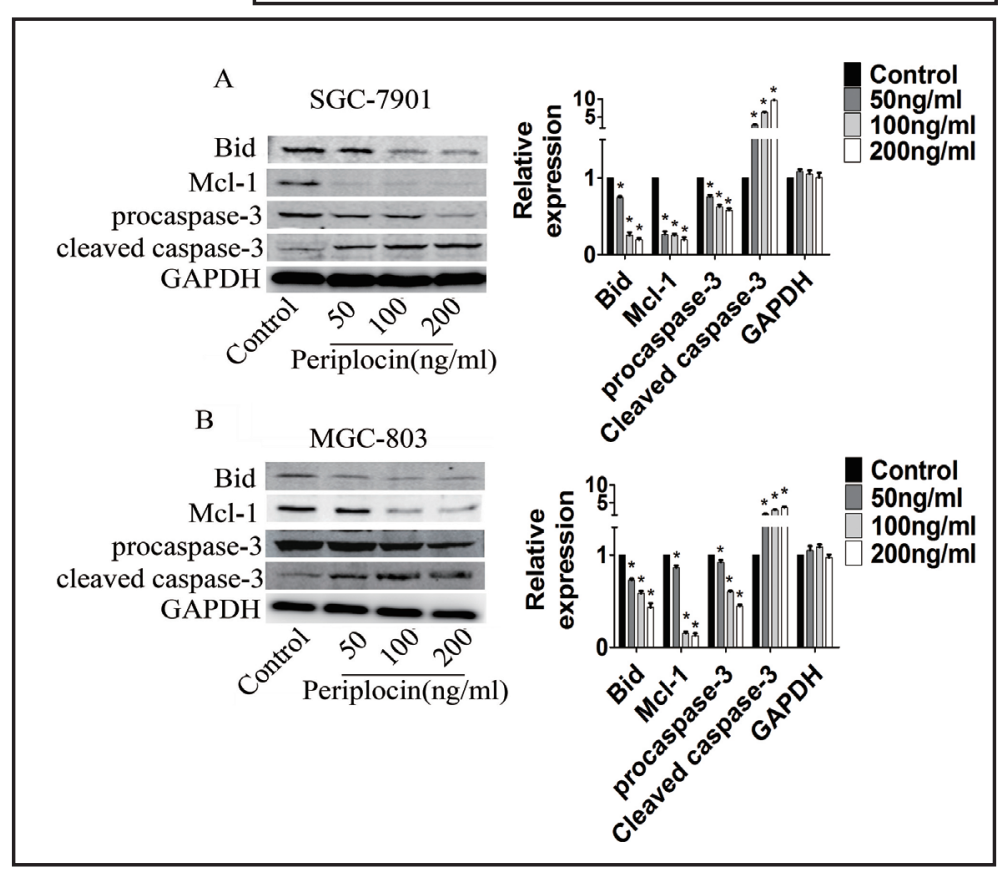

